\title{
ENSEMBLES DE RIESZ
}

\author{
VALÉRIE TARDIVEL
}

\begin{abstract}
Let $G$ be an abelian countable discrete group. We show that there exists no positive characterization of Riesz subsets of $G$, by proving that the Riesz subsets of $G$ form a coanalytic non-Borel subset of $2^{G}$.
\end{abstract}

Soit $G$ un groupe abélien discret dénombrable. Nous démontrons qu'il n'existe pas de caractérisation positive des ensembles de Riesz de $G$, en prouvant que les ensembles de Riesz de $G$ forment un coanalytique non borélien dans $2^{G}$.

Si $G$ est un groupe abélien discret dénombrable, le groupe dual $\hat{G}$ est un compact métrisable. Le spectre d'une mesure $\mu$, élément de $\mathcal{M}(\hat{G})$, est le support de $\hat{\mu}$ dans $G$ discret. Il est noté $S(\mu)$. On notera $m$ ou $d \xi$ la mesure de Haar sur $\hat{G}$. Les notions d'analyse de Fourier utilisées se trouvent dans les deux premiers chapitres du livre de Rudin [R1].

Soient $E$ et $F$, deux espaces polonais. Un ensemble $A \subset E$ est un ensemble analytique, s'il existe un borélien $B \subset E \times F$, tel que $A$ soit la projection de $B$ sur $E$. Le complémentaire d'un ensemble analytique est un ensemble coanalytique. Les résultats utilisés en théorie descriptive se trouvent dans Dellacherie [D].

On note $S$ l'ensemble des suites finies d'entiers. Pour $s$ et $s^{\prime}$ éléments de $S$, on note $s<s^{\prime}$ si $s$ est une section commençante de $s^{\prime}$. Si $s$ et $s^{\prime}$ sont deux éléments de $S$, on note $\widetilde{s s^{\prime}}$ la suite obtenue par concaténation des suites $s$ et $s^{\prime}$. On notera $|s|$ la longueur de $s$.

DEFINITION 1. On appelle arbre sur $\mathbf{N}$, un ensemble $T$ de suites finies d'entiers telles que $\left(n_{0}, n_{1}, \ldots, n_{k}\right) \in T \Rightarrow\left(n_{0}, n_{1}, \ldots, n_{k-1}\right) \in T . \quad\left(n_{k}\right)$ est une branche infinie pour $T$ si $\forall k\left(n_{0}, \ldots, n_{k}\right) \in T$. Un arbre $T$ est dit bien fondé, s'il ne contient aucune branche infinie.

$\mathrm{Si}$ on identifie un arbre $T$ avec sa fonction caractéristique, l'ensemble $T$ des arbres sur $\mathbf{N}$ est un sous-ensemble de $2^{S}$. On voit facilement que $\tau$ est un fermé de $2^{S}$. Si on le munit de la topologie induite par $2^{S}$, on obtient un compact métrisable.

On a le théorème suivant:

THEOREME 2 [D]. L'ensemble des arbres bien fondés sur $\mathbf{N}$ est un ensemble coanalytique non borélien.

Nous allons maintenant définir les ensembles de Riesz.

DEFINITION 3. Soit $G$ un groupe abélien discret dénombrable. Une partie $\Lambda \subset G$ est un ensemble de Riesz, si toute mesure définie sur $\hat{G}$, à spectre contenu dans $\Lambda$, est absolument continue par rapport à la mesure de Haar.

Received by the editors January 26, 1986.

1980 Mathematics Subject Classification. Primary 43A46, 04A15.

Key words and phrases. Riesz sets, coanalytic sets, harmonic analysis on compact abelian groups. 
REMARQUES. (1) L'ensemble vide est un ensemble de Riesz car $S(\mu)=\varnothing$ entraine $\mu=0$.

(2) Toute sous-partie d'un ensemble de Riesz est encore un ensemble de Riesz.

(3) D'après le théorème de $\mathbf{F}$. et $\mathbf{M}$. Riesz, $\mathbf{N}$ est un ensemble de Riesz dans $\mathbf{Z}$ [R2].

On appelle $\tau$ la topologie induite sur $G$ par le compactifié de Bohr $b G$ de $G[\mathbf{R 1}$ ].

Le théorème suivant, est une généralisation d'un résultat dû à Meyer $[\mathbf{M}]$.

THEOREME 4. Soit $E \subset G$ un ensemble de Riesz. Soit $\Lambda \subset G$, possédant la propriété suivante: pour tout $x \notin E$, il existe un $\tau$-voisinage ouvert $V_{x}$ de $x$ tel que $V_{x} \cap \Lambda$ soit un ensemble de Riesz. Alors $\Lambda$ est un ensemble de Riesz.

Demonstration. Soit $\mu$, un élément de $\mathcal{M}(\hat{G})$, dont le spectre est contenu dans $\Lambda$. Nous allons montrer que la partie singulière $\mu_{s}$, de $\mu$, est nulle. Soit $x \notin E$ et soit $V_{x}$ un $\tau$-voisinage ouvert de $x$ tel que $V_{x} \cap \Lambda$ soit un ensemble de Riesz. Il existe alors une mesure atomique $\sigma$ telle que $\hat{\sigma}(x)=1$ et $\hat{\sigma}=0$ sur le complémentaire de $V_{x}[\mathbf{M}]$. Alors, le spectre de $\mu * \sigma$ est contenu dans $V_{x} \cap \Lambda$, qui est un ensemble de Riesz. On en déduit que $(\mu * \sigma)_{s}=0$. Puisque $(\mu * \sigma)_{s}=\mu_{s} * \sigma[\mathbf{M}]$, en passant aux transformées de Fourier, on obtient $\hat{\mu}_{s}(x)=0$. Donc, le spectre de $\mu_{s}$ est contenu dans $E$, qui est ensemble de Riesz. On en déduit que $\mu_{s}$ est nulle, et que $\Lambda$ est un ensemble de Riesz.

Proposition 5. Tout ensemble fini est un ensemble de Riesz.

Demonstration. Soit $E=\left\{x_{0}, \ldots, x_{k}\right\}$ et soit $\mu$ une mesure dont le spectre est contenu dans $E$. La mesure $\nu$ définie par $\nu=\sum_{j=0}^{k} \hat{\mu}\left(x_{j}\right)\left(\xi, x_{j}\right) d \xi$ est telle que $\hat{\nu}=\hat{\mu}$. Donc $\mu=\nu$ et, par conséquent $\mu \ll m$.

DEFINITION 6. Soit $\left(x_{k}\right)$ une suite d'éléments de $G$. $\left(x_{k}\right)$ est dite dissociée si tout élément de $G$ admet au plus une représentation de la forme

$$
\sum_{j=0}^{n} \varepsilon_{k} x_{k} \quad \text { où } \varepsilon_{k} \in \begin{cases}\{-1,0,1\} & \text { si } 2 x_{k} \neq 0 \\ \{0,1\} & \text { si } 2 x_{k}=0\end{cases}
$$

THEOREME 7. Soit $\left(x_{k}\right)$ une suite dissociée d'éléments de G. Soit

$$
E=\left\{\sum_{k \geq 0} \varepsilon_{k} x_{k} \mid \varepsilon_{k}=-1,0,1 \text { si } 2 x_{k} \neq 0, \varepsilon_{k}=0,1 \text { si } 2 x_{k}=0\right\} \text {. }
$$

Alors E n'est pas un ensemble de Riesz.

Demonstration. Soit $\left(\alpha_{k}\right)$ une suite de réels compris entre $-\frac{1}{2}$ et $\frac{1}{2}$, et ne tendant pas vers 0 à l'infini. Pour tout $k$ élément de $\mathbf{N}$, on définit la fonction $P_{k}$ par

$$
P_{k}(\xi)= \begin{cases}\left(1+\alpha_{k}\left\langle x_{k}, \xi\right\rangle+\alpha_{k}\left\langle\overline{x_{k}, \xi}\right\rangle\right) & \text { si } 2 x_{k} \neq 0 \\ \left(1+\alpha_{k}\left\langle x_{k}, \xi\right\rangle\right) & \text { si } 2 x_{k}=0 .\end{cases}
$$

Pour tout $n$ élément de $\mathbf{N}$, on définit la mesure de probabilité $\mu_{n}$ par

$$
\mu_{n}=\prod_{k=0}^{n} P_{k}(\xi) d m(\xi)
$$


$\hat{\mu}_{n}$ vérifie alors

$$
\begin{cases}\hat{\mu}_{n}(0)=1 & \\ \hat{\mu}_{n}(x)=\prod_{k=0}^{j} \alpha_{k}^{\left|\varepsilon_{k}\right|} & \text { si } x=\sum_{k=0}^{j} \varepsilon_{k} x_{k}, j \leq n, \\ \hat{\mu}_{n}=0 & \text { sinon. }\end{cases}
$$

D'après (1), et puisque les mesures $\mu_{n}$ sont des mesures de probabilité, la suite $\left(\mu_{n}\right)_{n \geq 0}$ possède une limite vague $\mu$ telle que

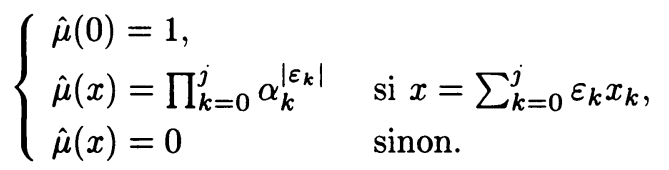

$\mu$ est une mesure de probabilité dont le support est contenu dans $E$. Puisque la suite $\left(\alpha_{k}\right)$ ne tend pas vers $0, \mu$ n'est pas absolument continue par rapport à la mesure de Haar. $E$ n'est donc pas un ensemble de Riesz.

Notre but, maintenant, est de montrer que $R(G)$, l'ensemble des ensembles de Riesz, n'est pas borélien (Théorème 10). En utilisant les Théorèmes 4 et 7 , nous allons associer à tout arbre bien fondé un ensemble de Riesz, et, à tout arbre qui n'est pas bien fondé, un ensemble qui n'est pas un ensemble de Riesz. Nous aurons à établir deux résultats préliminaires. Tout d'abord, nous introduisons sur $G$ une nouvelle topologie.

$G$ est un groupe abélien discret dénombrable. Le compactifié de Bohr de $G$, $b G$, est un compact, qui généralement n'est pas métrisable. Cependant, on peut trouver un compact métrisable $G^{*}$ contenant $G$. En effet, considérons dans $\hat{G}$, un sous-groupe dénombrable séparant les points de $G$. Son groupe dual, $G^{*}$, est un compact métrisable contenant $G$. En fait, $G^{*}$ peut être identifié à un quotient de $b G[\mathbf{R 1}] ; b G$ se projette donc sur $G^{*}$. Si on note $\tau^{*}$, la topologie induite sur $G$ par $G^{*}$, tout ouvert de $G$ pour $\tau^{*}$ est un ouvert de $G$ pour $\tau$.

LEMME 8. Soit $K$ un compact métrisable. Soient $D$ un ensemble dénombrable contenu dans $K$ et $F$ un fermé disjoint de $K$. Alors, il existe des ouverts $U_{n}$, deux à deux disjoints, tels que

(i) $\bar{U}_{n} \cap F=\varnothing$,

(ii) $\bigcup_{n=1}^{\infty} U_{n} \supset D$,

(iii) $U_{n} \cap D$ est ouvert et fermé dans $D$.

Demonstration. Soit $x$ un élément de $D$. $D$ étant dénombrable, on peut trouver $r \in \mathbf{R}^{*+}$ tel que $B(x, r) \cap D$ soit ouvert et fermé dans $D$, et $\bar{B}(x, r) \cap F=\varnothing$. On en déduit un recouvrement de $D$ par des boules $B_{n}$ telles que $B_{n} \cap D$ soit ouvert et fermé dans $D$, et $B_{n} \cap F=\varnothing$. Si l'on pose $U_{n}=B_{n} \backslash \bigcup_{k<n} \bar{B}_{k}$, les $U_{n}$ vérifient les conditions (i), (ii), et (iii) de l'énoncé.

THEOREME 9. Soit $G$ un groupe abélien discret dénombrable. Soit $G^{*}$, un compact métrisable, contenant $G$, et induisant sur $G$ la topologie $\tau^{*}$. Soit $\left(x_{k}\right)_{k \geq 0}$, une suite d'éléments non nuls de $G$, tendant vers 0 pour la topologie $\tau^{*}$.

Alors, pour tout $s \in S$, on peut construire:

(i) un ensemble

$$
E_{s}=\left\{\sum_{k=0}^{|s|-1} \varepsilon_{k} t_{k}^{(s)} \mid \varepsilon_{k}=-1,0,1 \text { si } 2 t_{k}^{(s)} \neq 0, \varepsilon_{k}=0,1 \text { si } 2 t_{k}^{(s)}=0, t_{k}^{(s)} \in\left(x_{k}\right)\right\}
$$

tel que $s<s^{\prime} \Rightarrow E_{s} \subset E_{s^{\prime}}$ 
(ii) une partition $\left(W_{j}^{(s)}\right)_{j \in \mathbf{N}}$ en ouverts fermés, pour la topologie induite par $G^{*}$, de $G \backslash E_{s}$;

(iii) si $s=\overparen{r k}$, un ouvert $U_{s}$ de $G \backslash E_{r}$, pour la topologie induite par $G^{*}$, réunion d'éléments de $\left(W_{j}^{(r)}\right)$ et vérifiant:

$$
\begin{array}{cc}
U_{\widetilde{r k}} \cap U_{\widehat{r n}}=\varnothing \quad \text { si } k \neq n, \\
E_{t} \backslash E_{r} \subset U_{\widehat{r k}} \quad \text { si } \widehat{r k}<t .
\end{array}
$$

Demonstration. On note $\left(p_{k}\right)_{k \geq 0}$ la suite des nombres premiers. A toute suite finie $s=\left(n_{0}, \ldots, n_{k}\right)$, on associe l'entier naturel $\nu(s)=p_{0}^{n_{0}} p_{1}^{n_{1}} \cdots p_{k}^{n_{k}+1}$. On vérifie aisément, que $\nu$ est une bijection de $S$ sur $\mathrm{N}$, vérifiant $s<s^{\prime} \Rightarrow \nu(s) \leq \nu\left(s^{\prime}\right)$. Nous allons construire, par récurrence, pour tout $k=\nu(u)$ un ensemble $E_{u}$, et des parties finies $A_{\overrightarrow{s n}}^{k}$ de $\mathbf{N}$ tels que

$(\alpha)$ si $k^{\prime}>k, A_{\stackrel{s n}{k^{\prime}}}^{k^{\prime}} \supset A_{\stackrel{s n}{k}}^{k}$,

( $\beta)$ si $m \neq n, A_{s m}^{k n} \cap A_{s n}^{k n}=\varnothing$,

$(\gamma)$ si $t>\widehat{s n}$ et $\nu(t) \leq k, E_{t} \backslash E_{s} \subset \bigcup_{j \in A_{s n}^{k}} W_{j}^{(s)}$,

$(\delta)$ si $\nu(\widehat{s n})>k, A_{s n}^{k}=\varnothing$.

On pose $E_{\varnothing}=\{0\}$. Grâce au Lemme 8 , on construit $\left(W_{j}^{\varnothing}\right)_{j \in \mathbf{N}}$ vérifiant (ii). A cette étape de la construction, il n'y a que la condition $(\delta)$ qui est vérifiée. Supposons la construction effectuée pour tout $k<n=\nu(s)$, et effectuons la construction pour s. On a donc construit:

-des ensembles $E_{u}$, pour $\nu(u)<\nu(s)$, vérifiant (i),

-des partitions $\left(W_{j}^{(u)}\right)_{j \in \mathbf{N}}$, pour $\nu(u)<\nu(s)$, vérifiant (ii),

-des parties finies de $\mathbf{N}, A_{\widetilde{u p}}^{k}$, vérifiant $(\alpha),(\beta),(\gamma)$, et $(\delta)$.

On a $s=\widehat{r m}$, par exemple. Il faut trouver $t_{|r|}^{(s)}$, élément de $G \backslash E_{r}$ et extrait de la suite $\left(x_{k}\right)_{k \geq 0}$, pour construire

$$
E_{s}=E_{r} \cup\left\{E_{r}+t_{|r|}^{(s)}\right\} \cup\left\{E_{r}-t_{|r|}^{(s)}\right\} \quad \text { si } 2 t_{|r|}^{(s)} \neq 0
$$

ou

$$
E_{s}=E_{r} \cup\left\{E_{r}+t_{|r|}^{(s)}\right\} \quad \text { si } 2 t_{|r|}^{(s)}=0
$$

et il faut construire les $A_{\frac{u p}{n}}^{n}$ vérifiant $(\alpha),(\beta),(\gamma)$, et $(\delta)$.

Pour simplifier les notations, posons $t_{|r|}^{(s)}=t$. Soit $x$ élément de $E_{r}, x \neq 0$. Il existe une suite $u$, de longueur maximale, pour laquelle on a: $x \in E_{r} \backslash E_{u} \subset$ $\bigcup_{j \in A_{u p}^{n-1}} W_{j}^{(u)}$ si $\widehat{u p}<r(*)$. Nous allons trouver $t$ et $A_{u p}^{n}$ tels que

(a) pour tout $x$ vérifiant $(*)$

$$
\begin{array}{ll}
x+t \in \bigcup_{j \in A_{\widehat{u p}}^{n}} W_{j}^{(u)} & \text { si } \widehat{u p}<r, \\
x-t \in \bigcup_{j \in A_{\frac{u p p}{n}}^{n}} W_{j}^{(u)} & \text { si } \widehat{u p}<r,
\end{array}
$$


la deuxième condition pouvant être nulle

(b)

$$
t \in \bigcup_{j \in A_{\text {up }}^{n}} W_{j}^{(u)}, \quad-t \in \bigcup_{j \in A_{u}^{n}} W_{j}^{(u)},
$$

la deuxième condition pouvant être nulle.

D'après la condition $(\delta)$, il suffit de construire les $A_{\widetilde{u p}}^{n}$ vérifiant $(\alpha),(\beta)$ et $(\gamma)$ pour $\nu(\widehat{u p}) \leq n$.

La suite $\left(x_{k}\right)$ tend vers 0 , donc, pour tout $x$ vérifiant $(*)$, la suite $x+t$, où $t \in\left(x_{k}\right)$, tend vers $x$ (il en est de même pour la suite $x-t$ ). Si $x \in \bigcup_{j \in A_{u p}^{n-1}} W_{j}^{(u)}$, pour $t$ suffisamment grand, puisque $\bigcup_{j \in A_{u p}^{n-1}} W_{j}^{(u)}$ est ouvert, en peut avoir $(x+t)$, (respectivement $x-t$ ) éłément de $\bigcup_{j \in A_{u p}^{n-1}} W_{j}^{(u)}$. Puisqu'il n'y a qu'un nombre fini de tels $x$, on peut trouver $t$ tel que (a) soit vérifié, dès que $A_{u p}^{n} \supset A_{u p}^{n-1}$.

Pour que la condition (b) soit vérifiée, il faut que l'on puisse trouver $t$ dans la suite $\left(x_{k}\right)$ tel que pour tout $\widehat{u p}<s, t$ soit dans un $W_{j(\widehat{u p})}^{(u)}$ et $-t$ soit dans un $W_{j^{\prime}(\widehat{u p})}^{(u)}$ qui n'aient pas déjà été choisis. Il suffit alors de poser $A_{\widetilde{u p}}^{n}=A_{\widetilde{u p}}^{n-1} \cup\left\{j(\widetilde{u p}), j^{\prime}(\widetilde{u p})\right\}$ pour avoir le résultat, puisque les $W_{j}^{(u)}$ forment des partitions de $G \backslash E_{u}$.

Pour chaque $u$, telle que $\nu(u)<\nu(s)$, il n'y a qu'un nombre fini de $W_{j}^{(u)}$ qui ont été choisis. Supposons, qu'à partir d'un certain rang, tous les éléments de la suite $\left(x_{k}\right)$ soient dans la réunion de ces $W_{j}^{(u)}(\nu(u)<\nu(s))$. Alors, il existe un $W_{j}^{(u)}$ contenant un nombre infini d'éléments de la suite $\left(x_{k}\right)$. Donc, il existe une suite extraite qui se trouve dans l'un des $W_{j}^{(u)}$. Cette suite extraite converge vers 0 , et, $W_{j}^{(u)}$ étant fermé, doit contenir cette limite. Or, ceci est impossible par construction de $W_{j}^{(u)}$. Il y a donc une infinité d'éléments de $\left(x_{k}\right)$ en dehors des $W_{j}^{(u)}$ déjà choisis. S'il y a une infinité d'éléments de la suite $\left(x_{k}\right)$ qui ne sont pas d'ordre 2, un raisonnement analogue au précédent montre qu'il y a une infinité d'éléments $t$ de la suite tels que $t$ et $-t$ soient en dehors des $W_{j}^{(u)}$ déjà choisis. On choisit donc $t, j(\widehat{u p})$ et $j^{\prime}(\widehat{u p})$, pour $\widehat{u p}<s$ tels que la condition (b) soit satisfaite. On achève ainsi la construction de $E_{s}$, et des $A_{u p}^{n}$ vérifant $(\alpha),(\beta),(\gamma)$, et $(\delta)$ au rang $n . E_{s}$ vérifie (i). Ensuite, le Lemme 8 permet de construire la partition $\left(W_{j}^{(s)}\right)_{j \in \mathbf{N}}$ vérifiant (ii). Il suffit alors de poser $U_{\widehat{s n}}=\bigcup\left\{W_{j}^{(s)} \mid j \in \bigcup_{k} A_{s n}^{k}\right\}$ pour que (iii) soit vérifiée.

Ceci achève la démonstration du théorème.

THEOREME 10. Soit $G$ un groupe abélien discret dénombrable. $R(G)$ n'est pas borélien.

DEMONSTRATION. Soit $\left(t_{k}\right)$ une suite d'éléments non nuls de $G$ tendant vers 0 pour $\tau^{*}$. On peut, par induction, à partir de la suite $\left(t_{k}\right)$, construire une suite dissociée $\left(x_{k}\right)$ tendant vers 0 pour $\tau^{*}$ [G-McG, Lemma 7.1.1] A partir de cette suite $\left(x_{k}\right)$, on effectue la construction donnée par le Théorème 9 . 
Soit $T$ un arbre sur $\mathbf{N}$. Posons $F(T)=\bigcup_{s \in T} E_{s}$.

(a) Nous allons montrer: $F(T)$ est un ensemble de Riesz si, et seulement si, $T$ est bien fondé.

(1) Si $T$ admet une branche infinie, $F(T)$ contient un ensemble de la forme $\left\{\sum_{k \geq 0} \varepsilon_{k} x_{k}^{\prime} \mid \varepsilon_{k}=-1,0,1\right.$ si $2 x_{k}^{\prime} \neq 0, \varepsilon_{k}=0,1$ si $\left.2 x_{k}^{\prime}=0\right\}$ où la suite $\left(x_{k}^{\prime}\right)$ est une suite dissociée, extraite de la suite $\left(x_{k}\right)$. D'après le Théorème $7, F(T)$ n'est pas un ensemble de Riesz.

(2) Supposons que $F(T)$ ne soit pas un ensemble de Riesz. Pour $s \in S$, on pose $F_{s}(T)=\bigcup_{u>s} E_{u}$.

D'après la construction effectuée au théorème précédent, on a

$$
F_{s}(T) \subset E_{s} \cup\left(\bigcup_{n \in \mathbb{N}} U_{\widehat{s n}}\right) .
$$

Nous allons montrer que $T$ contient une branche infinie. On a

$$
F(T)=\bigcup_{n} F_{\langle n\rangle}(T)
$$

Montrons qu'il existe $\left\langle n_{0}\right\rangle \in T$, telle que $F_{\left\langle n_{0}\right\rangle}(T)$ ne soit pas un ensemble de Riesz. Supposons que ceci soit faux. Soit $x \in G \backslash E_{\varnothing} . x$ est dans l'un des $W_{j}^{(\varnothing)}$, soit $W_{j(x)}^{(\varnothing)}$. D'après la construction effectuée au Théorème $9, W_{j(x)}^{(\varnothing)} \cap F(T)=\varnothing$ ou, il existe $\langle n\rangle$ tel que $j(x) \in \bigcup_{k} A_{n}^{k}$ et $W_{j(x)}^{(\varnothing)} \cap F(T) \subset F_{\langle n\rangle}(T)$. On en déduit que $W_{j(x)}^{(\varnothing)} \cap F(T)$ est un ensemble de Riesz. Puisque les $W_{j(x)}^{(\varnothing)}$ sont des ouverts de $G$ pour $\tau$ et $E_{\varnothing}$ est un ensemble de Riesz, d'après le Théorème $4, F(T)$ est un ensemble de Riesz. Il existe donc bien $n_{0}$ telle que $F_{\left\langle n_{0}\right\rangle}(T)$ ne soit pas un ensemble de Riesz. De plus, $\left\langle n_{0}\right\rangle \in T$ car sinon $F_{\left\langle n_{0}\right\rangle}(T)$ serait l'ensemble vide, donc un ensemble de Riesz.

Supposons qu'il existe $s \in T$ telle que $F_{s}(T)$ ne soit pas un ensemble de Riesz, et montrons que l'on peut trouver $n \in \mathrm{N}$, tel que $F_{\langle\hat{s n}\rangle}(T)$ ne soit pas un ensemble de Riesz et tel que $\langle\widehat{s n}\rangle \in T$. On a $F_{s}(T)=\bigcup_{n} F_{\langle\widehat{s n}\rangle}(T)$. Supposons, que, pour tout $n, F_{\langle\overline{s n}\rangle}(T)$ soit un ensemble de Riesz. Soit $x \in G \backslash E_{s} . x$ est donc dans l'un des $W_{j}^{(s)}$, soit $W_{j(x)}^{(s)}$. Comme précédemment, en utilisant le Théorème 9 , on montre que $W_{j(x)}^{(s)} \cap F_{s}(T)$ est un ensemble de Riesz. Les $W_{j(x)}^{(s)}$ étant des ouverts de $G$ pour la topologie $\tau$ et $E_{s}$ étant un ensemble de Riesz, une nouvelle utilisation du Théorème 4 montre que $F_{s}(T)$ est un ensemble de Riesz. Donc, il existe bien $n$ tel que $F_{\langle\overline{s n}\rangle}(T)$ ne soit pas un ensemble de Riesz. Puisque $F_{\langle\overline{s n}\rangle}(T)$ ne peut pas être vide, $\langle\overparen{s n}\rangle \in T$.

Donc, pour toute suite finie $s \in T$ telle que $F_{s}(T)$ ne soit pas un ensemble de Riesz, il existe $n \in \mathrm{N}$ tel que $\left\langle\widehat{s n\rangle} \in(T)\right.$, et $F_{\langle\widehat{s n}\rangle}(T)$ ne soit pas un ensemble de Riesz.

On en déduit l'existence d'une suite infinie d'entiers $\sigma$, telle que, pour toute section commençante $s$ de $\sigma, F_{s}(T)$ soit non vide et ne soit pas un ensemble de Riesz et $s \in T . \sigma$ est donc une branche infinie pour $T$.

Ceci achève la partie (a) de la démonstration.

(b) On montre facilement que l'application $F$ de $T$ dans $2^{G}$ définie par $F(T)=$ $\bigcup_{s \in T} E_{s}$, est de première classe. 
Si $R(G)$ était borélien, son image réciproque par $F$ serait un borélien de $\tau$. Or, cette image réciproque est l'ensemble des arbres bien fondés sur $\mathbf{N}$ qui, d'après le Théorème 2, n'est pas borélien. Donc $R(G)$ n'est pas borélien.

THEOREME 11. Soit $G$ un groupe abélien discret dénombrable. $R(G)$ est un ensemble coanalytique dans $2^{G}$.

Demonstration. $2^{G}$ est un espace polonais. $\mathcal{M}^{1}(\hat{G})$, muni de la topologie vague, est également un espace polonais.

Or, $\Lambda \subset G$ est un ensemble de Riesz, si et seulement si,

$$
\forall \mu \in \mathcal{M}^{1}(\hat{G}) \quad(\forall x \in G(x \notin \Lambda \Rightarrow \hat{\mu}(x)=0)) \Rightarrow \mu \ll m
$$

or, $\mu$ est absolument continue par rapport à $m$ si, et seulement si, $|\mu|$ est absolument continue par rapport à $m$, c'est-à-dire si, et seulement si

$$
\forall \varepsilon>0 \quad \exists \eta>0 \quad \forall B \text { borélien } \quad m(B)<\eta \Rightarrow|\mu|(B)<\varepsilon
$$

ou encore

$$
\forall k>0 \quad \exists p>0 \quad \forall B \text { borélien } \quad m(B)<\frac{1}{p} \Rightarrow|\mu|(B)<\frac{1}{k} .
$$

De plus, il suffit de faire la vérification sur les réunions finies d'ouverts de base. Ces réunions sont dénombrables car $\hat{G}$, étant un compact métrisable, admet une base dénombrable.

Soit $\left(U_{n}\right)_{n \in \mathrm{N}}$ l'ensemble de ces réunions finies d'ouverts de base. $\Lambda \subset G$ est un ensemble de Riesz si, et seulement si,

$$
\begin{aligned}
\forall \mu \in \mathcal{M}^{1}(\hat{G}) \quad(\forall x \in G(x \notin \Lambda \Rightarrow \hat{\mu}(x)=0)) \\
\quad \Rightarrow\left(\forall k>0 \exists p>0 \forall n m\left(U_{n}\right)<\frac{1}{p} \Rightarrow|\mu|\left(U_{n}\right)<\frac{1}{k}\right) .
\end{aligned}
$$

Considérons le borélien suivant de $\mathcal{M}^{1}(\hat{G}) \times 2^{G} \times 2^{G}$ :

$$
\begin{aligned}
B=\bigcup & \bigcap_{x \in G} \bigcup_{k>0} \bigcap_{p>0}\left(\{\mu \mid \hat{\mu}(\gamma) \neq 0\} \times\{\Lambda \mid x \notin \Lambda\} \times 2^{G} \cup \mathcal{M}^{1}(\hat{G}) \times 2^{G}\right. \\
& \left.\times\left\{U_{n} \mid m\left(U_{n}\right) \geq \frac{1}{p}\right\} \cup\left\{\mu|| \mu \mid\left(U_{n}\right)<\frac{1}{k}\right\} \times 2^{G} \times 2^{G}\right) .
\end{aligned}
$$

Le complémentaire de $B, B^{c}$, est un borélien de $\mathcal{M}^{1}(\hat{G}) \times 2^{G} \times 2^{G}$. La projection de $B^{c}$ sur le deuxième facteur de $\mathcal{M}^{1}(\hat{G}) \times 2^{G} \times 2^{G}$ est un ensemble analytique de $2^{G}$, soit $A$. Or, $R(G)=A^{c}$, donc $R(G)$ est coanalytique dans $2^{G}$.

COROLlaIRE 12. Si $G$ est un groupe abélien discret dénombrable, $R(G)$ est un ensemble coanalytique non borélien dans $2^{G}$.

Je remercie vivement Monsieur le Professeur Jean Saint Raymond pour le temps qu'il a bien voulu m'accorder, et tout l'intérêt qu'il a porté à la réalisation de ce travail.

Je tiens à remercier également Monsieur le Professeur Gilles Godefroy pour les indications qu'il m'a données sur les ensembles de Riesz. 


\section{BIBLIOGRAPHIE}

[D] C. Dellacherie, Ensembles analytiques: théorèmes de séparation et applications, Séminaire de Probabilités IX, Lecture Notes in Math., vol. 465, Springer-Verlag, Berlin and New York, 1975, pp. 336-372.

[G-McG] C. G. Graham and O. C. McGehee, Essays in commutative harmonic analysis, SpringerVerlag, New York and Berlin, 1979.

[M] Y. Meyer, Spectres des mesures et mesures absolument continues, Studia Math. 30 (1968), 87-99.

[R1] W. Rudin, Fourier analysis on groups, Interscience Tracts, no. 12, Wiley, New York, 1962.

[R2] _ Real and complex analysis, 2nd ed., McGraw-Hill, New York, 1974.

Equipe d'Analyse, Université Paris 6, 4, Place Jussieu T/46/0 - 4̇̀me Étage, 75256 PARIS CEDEX 05, FRANCE 